\title{
An objective interpolation method for spatiotemporal distribution of marine plankton
}

\author{
Meng Zhou* \\ Scripps Institution of Oceanography, University of California, San Diego, La Jolla, California 92093-0202, USA
}

\begin{abstract}
A Lagrangian-Eulerian interpolation method for the objective analysis of marine plankton data was developed. Based on the Gauss-Markov theorem, this method takes into account the advection effect on the distribution of passive marine plankton, and yields an estimate and confidence at every interpolating point $(x, y, z, t)$ which is optimal in the least squares error This method was demonstrated in the analysis of plankton data collected in the California Current region during June 1993. The interpolated time series of spatial distributions of plankton revealed areas where plankton features were more permanent due to weak advection and areas where plankton features were more timedependent due to the existence of strong currents. Results show north-south transports and exchanges of plankton populations produced by the complex flow system in the California Current region. This Lagrangian-Eulerian interpolation produces synoptic spatial distributions of plankton at given times and their error fields, and can be used as a basic analytical tool to understand advection effects in plankton distributions.
\end{abstract}

KEY WORDS: Objective analysis - Interpolation - Spatiotemporal variation . Plankton distribution . Advection - California Current

\section{INTRODUCTION}

The distribution of plankton in oceans has been found to be very patchy and temporal (Steele \& Henderson 1992, Huntley \& Niiler 1995). For example, the spatial scale of zooplankton in the California Current region is on the order of $50 \mathrm{~km}$ (Huntley et al. 1995), which is mostly determined by physical factors, such as circulation, mixing, and current convergence. The time scale (approximately $10^{\circ}$ to $10^{2} \mathrm{~d}$ ) is determined by the generation time and the advection-dispersion processes of zooplankton, excluding the long term seasonal and interannual variabilities. These spatial and temporal variabilities have made it very difficult to conduct a synoptic mesoscale survey because the time required to conduct the survey is limited by the speed of our current sampling technology.

\footnotetext{
- Present address: Large Lakes Observatory, University of Minnesota, 10 University Dr., Duluth, Minnesota 55812, USA

E-mail: mzhou@d.umn.edu
}

The conceptual survey error can be illustrated by the ratio of the survey resolution $L_{5}$ to the spatial scale of plankton distribution $L_{z}$ and the ratio of the survey time $T_{s}$ to the time scale of plankton distribution $T_{z}$. These ratios represent the relative errors of sampling in space and time respectively, i.e.

$$
\frac{L_{s}}{L_{z}}, \frac{T_{s}}{T_{z}}
$$

Increasing the spatial survey resolution, which can reduce the spatial sampling error, unfortunately re. quires more survey time and unavoidably increases the sampling error over time. An optimal survey design in both spatial and temporal resolutions is necessary for obtaining synoptic plankton distributions in physical space and time from samples.

In practice, a spatial survey provides a sequence of samples in space and time. A direct map is meaningful only if the survey time is much shorter than the distribution's temporal variation. As this rarely happens the blanks in space and time often need to be filled by interpolation or even extrapolation for different analyt- 
ical purposes. The choice of interpolation methods is arbitrary. A well known method is 'Kriging' of 2- or 3-dimensional spatial interpolation (Davis 1986, Isaaks \& Srivaslava 1989, Dentsch \& Journel 1992). When the spatial distribution of a variable is anisotropic and time-dependent, the task of interpolation becomes more difficult.

Any interpolation method implies a number of assumptions and a certain degree of smoothness of the measured fields. Objective analysis based on the Gauss-Markov theorem explicitly shows the result in statistical estimation theory (Liebelt 1967). Based on statistical assumptions, this method yields an estimate at every point $(\mathbf{x}, t)$ which is optimal in the least squares error. The analysis of error fields can be made to guide the survey design in space and time. This method was first applied in meteorology by Gandin (1965) for the objective analysis of pressure and wind fields, and weather prediction. Later it was used in oceanographic studies by Bretherton et al. (1976), Clancy (1983), Mooers \& Robinson (1984), and Carter \& Robinson (1987).

In these applications of objective analysis, estimates rely on Eulerian correlation or covariance functions $C\left(\mathbf{x}_{2}, \mathbf{x}_{1}, t_{2}, t_{1}\right)$, where $\mathbf{x}_{1}$ and $\mathbf{x}_{2}$ are the spatial positions and $t_{1}$ and $t_{2}$ are the times. In the analysis of eddy fields, the correlation function can include the translation speed of eddies (Mooers \& Robinson 1984, Carter \& Robinson 1987), i.e. $C\left[\mathbf{x}_{2}-\mathbf{x}_{1}+\mathbf{c}\left(t_{2}-t_{1}\right)\right]$ where $\mathbf{c}$ is the translation velocity. The estimate at the point $(\mathbf{x}, t)$ will be poor if the correlation between this point and the surrounding sampling points is low, where $\mathbf{x}$ is the location and $t$ is the time, respectively.

Marine plankton can be considered as passive particles in the first approximation. The advection of plankton by currents reduces the Eulerian correlation between 2 points in space and time. To reduce the advection effect, the Eulerian correlation function can include the advection processes, such as $C\left[\mathbf{x}_{2}-\mathbf{x}_{1}+\mathbf{u}\left(t_{2}-t_{1}\right)\right]$ where $\mathbf{u}$ is the current velocity. In general, the patch scale of plankton is smaller than the eddy field. Mean current is greater than the eddy translation speed (Huntley \& Niler 1995, Huntley et al. 1995). The Eulerian correlation between 2 points vanishes in a time period (decorrelation time scale) which can be obviously estimated by the ratio of the spatial scale $L_{z}$ of plankton patchiness to the velocity scale $U$, i.e. $L_{z} / U$. In the California Current region where $L_{z}=50 \mathrm{~km}$ and $U=20 \mathrm{~cm} \mathrm{~s}^{-1}$ (Huyer et al. 1994 , Huntley et al. 1995), the decorrelation time scale is on the order of $3 \mathrm{~d}$. This decorrelation time scale is the same as or shorter than the time needed to conduct the survey in a region of $100 \times 100 \mathrm{~km}^{2}$ at a spatial resolution of $10 \mathrm{~km}$. Such spatial resolution is necessary to resolve the distribution of zooplankton at the meso- scale of $50 \mathrm{~km}$. Thus, objective analysis based on the Eulerian correlation will indicate low-confidence estimates for those blanks in space and time.

Estimating the spatial distribution of plankton at a given time allows for a more familiar understanding of plankton processes. In the vicinity of the California Current, observations show that plankton distribution is closely related to physical features such as circulation, eddies, and upwelling areas (Huntley et al. 1995). During our 1993 surveys in this area, high plankton abundance and biomass maximums were found in both cyclonic and anticyclonic eddies, and minimums in nearshore and offshore regions. However, the plankton distribution was time-dependent, especially in regions where strong currents existed. The time scales of these maximums and minimums were not necessary longer than or the same as the time scales of physical features. Understanding the coupled physical-biological processes is probably best accomplished by first reconstructing a synoptic spatial distribution of plankton from samples including advective effects.

How can we obtain a statistical meaningful synoptic distribution of plankton from samples? Here a Lagrangian-Eulerian objective interpolation technique is introduced that minimizes effects of advection on the decorrelation time scales and optimizes the mean square error.

This Lagrangian-Eulerian objective interpolation technique is demonstrated by using the plankton data obtained by an Optical Plankton Counter (OPC, Focal Instruments, Dartmouth, NS, Canada) in the California Current region. The results provide insight into the time-dependent distribution of zooplankton relative to the California Current system. The primary objective is to test this method, not to conduct a systematic analysis of all physical and biological data. Therefore this approach should be considered as experimental, and the results as suggestive rather than conclusive.

\section{BASIC THEORY}

We briefly review the fundamental technique of objective analysis. Following Bretherton et al. (1976). we assume that there are a limited number of samples of a scale field $\theta$ at $\left(\mathbf{x}_{11}, t_{i}\right)(i=1, \ldots, N)$. We assume the measurement $\varphi$ is the true value $\theta$ plus a random error $\varepsilon$, expressed as

$$
\varphi\left(\mathbf{x}_{n} t_{i}\right)=\theta\left(\mathbf{x}_{n} t_{i}\right)+\varepsilon\left(\mathbf{x}_{1} t_{i}\right) \quad(i=1, \ldots N)
$$

Errors $\varepsilon$ are uncorrelated with each other and with the field $\theta$ but have known variance $E$, i.e.

$$
\begin{gathered}
\left\langle\varepsilon\left(\mathbf{x}_{1}, t_{i}\right) \theta\left(\mathbf{x}_{j,}, t_{j}\right)\right\rangle=0 \\
\left\langle\varepsilon\left(\mathbf{x}_{i}, t_{j}\right) \varepsilon\left(\mathbf{x}_{j}, t_{j}\right)\right\rangle=E \delta_{i j} \quad(i=1, \ldots, N)
\end{gathered}
$$


where $\langle\cdot\rangle$ is the ensemble average. The symbol $\delta_{i j}$ is called the Kroenecker delta and is defined by

$$
\begin{aligned}
\delta_{I J} & =1 & & \text { if } i=j \\
& =0 & & \text { if } i \neq j
\end{aligned}
$$

The estimate $\theta^{*}$ for $\theta$ at $(\mathbf{x}, t)$ is

$$
\theta^{*}(\mathbf{x}, t)=\sum_{i=1}^{N} w\left(\mathbf{x}, \mathbf{x}_{i}, t, t_{i}\right) \varphi\left(\mathbf{x}_{1}, t_{i}\right)
$$

where $w$ is the weight. From the least squares optimum, we have

$$
w\left(\mathbf{x}, \mathbf{x}_{i}, t, t_{i}\right)=\sum_{j=1}^{N} C\left(\mathbf{x}, \mathbf{x}_{j}, t_{i} t_{j}\right) A^{-1}\left(\mathbf{x}_{i}, \mathbf{x}_{j}, t_{i}, t_{j}\right)
$$

where $C\left(\mathbf{x}, \mathbf{x}_{i}, t, t_{i}\right)$ is the ensemble spatiotemporal correlation or covariance function, i.e.

$$
\begin{aligned}
C\left(\mathbf{x}, \mathbf{x}_{1}, t_{1} t_{j}\right) & =\left\langle\theta(\mathbf{x}, t) \theta\left(\mathbf{x}_{1}, t_{t}\right)\right\rangle \\
A\left(\mathbf{x}_{1}, \mathbf{x}_{j}, t_{j}, t_{j}\right) & =\left\langle\varphi\left(\mathbf{x}_{1}, t_{i}\right) \varphi\left(\mathbf{x}_{j}, t_{j}\right)\right\rangle \\
& =C\left(\mathbf{x}_{1}, \mathbf{x}_{j}, t_{2}, t_{j}\right)+E \delta_{i j}
\end{aligned}
$$

and $A^{-1}\left(\mathbf{x}_{i l}, \mathbf{x}_{j}, t_{j l}, t_{j}\right)$ is the inverse matrix of $A\left(\mathbf{x}_{i}, \mathbf{x}_{j}, t_{i,}, t_{j}\right)$. The error variance for $\theta^{*}$ is

$$
\begin{aligned}
& \varepsilon(\mathbf{x}, t)^{2}=C(\mathbf{x}, \mathbf{x}, t, t) \\
& +\sum_{i=1}^{N} \sum_{j=1}^{N} C\left(\mathbf{x}_{1}, \mathbf{x}_{1}, t_{1} t_{i}\right) C\left(\mathbf{x}_{1} \mathbf{x}_{j}, t_{1} t_{j}\right) A^{-1}\left(\mathbf{x}_{i}, \mathbf{x}_{j}, t_{i}, t_{j}\right)
\end{aligned}
$$

Here the relevant estimate can be made only if we can obtain the correct Eulerian spatiotemporal correlation defined by Eq. (7). This correlation is fundamentally a function of locations $\mathbf{x}$ and $\mathbf{x}^{*}$, and the times $t$ and $t^{*}$ in an inhomogeneous and anisotropic field, i.e.

$$
C\left(\mathbf{x}, \mathbf{x}^{*}, t, t^{*}\right)=R(\mathbf{x}, \mathbf{r}, t, \tau)
$$

where $\mathbf{r}=\mathbf{x}-\mathbf{x}^{*}=(\Delta x, \Delta y, \Delta z)$ is the distance between 2 locations, $\tau=t-t^{*}$ is the time lag, and $R$ is an arbitrary function. This correlation can be simplified when we have the field:

homogeneous

$$
C\left(\mathbf{x}, \mathbf{x}^{*}, t, t^{*}\right)=R(\mathbf{r}, t, \tau)
$$

homogeneous and stationary

$$
C\left(\mathbf{x}, \mathbf{x}^{*}, t, t^{*}\right)=R(\mathrm{r}, \tau)
$$

homogeneous and steady

$$
C\left(\mathbf{x}, \mathbf{x}^{*}, t, t^{*}\right)=R(\mathbf{r})
$$

homogeneous, steady and isotropic

$$
C\left(\mathbf{x}, \mathbf{x}^{*}, t, t^{*}\right)=R(|\mathbf{r}|)
$$

In most interpolation methods, the last assumption is applied. Unfortunately this kind of field is rarely found in the oceans. The ocean usually is vertically stratified and limited by the bottom topography, so that the vertical scale is much less than the horizontal scale. On the other hand, obtaining the ensemble correlation of Eq. (10) is impractical when using our current technology. Some as- sumptions and approximations have to be made in order to obtain the correlation function, e.g. the homogeneous and steady assumptions, by which the correlation function is independent of location and time. A commonly used form of correlation functions can be written as

$$
\begin{gathered}
C\left(\mathbf{x}, \mathbf{x}^{*}, t, t^{*}\right)=R(\mathbf{r}, \tau)=\left(1-r^{2}\right) \exp \left(-I^{2}\right) \\
r^{2}=\left(\frac{\Delta x}{L_{x}}\right)^{2}+\left(\frac{\Delta y}{L_{y}}\right)^{2}+\left(\frac{\Delta z}{L_{z}}\right)^{2}+\left(\frac{\tau}{T}\right)^{2}
\end{gathered}
$$

where $L_{x}, L_{Y^{\prime}} L_{z}$ are the spatial scales in $x, y, z$ directions, and $T$ is the time scale (Bretherton et al. 1976, Clancy 1983, Mooers \& Robinson 1984, Carter \& Robinson 1987).

In the California Current region, if we take the plankton patch scale of $50 \mathrm{~km}$, the horizontal diffusivity of $10^{5}$ to $10^{7} \mathrm{~cm}^{2} \mathrm{~s}^{-1}$ (Gill 1982, Paduan \& Niiler 1993), the current scale of $20 \mathrm{~cm} \mathrm{~s}^{-1}$ (Huntley et al. 1995), and the plankton population turnover time scale of $10^{1} \mathrm{~d}$ (Mullin 1991, Huntley et al. 1995), we have the diffusion time scale of $3 \times 10^{1}$ to $3 \times 10^{3} \mathrm{~d}$, the advection time scale of $3 \mathrm{~d}$, and the plankton turnover time scale of $10^{1} \mathrm{~d}$. The decorrelation time scale of plankton distributions is mostly determined by the advection. The survey in the entire region (Fig. 1; Huntley et al. 1995) took $21 \mathrm{~d}$. If we eliminate the effect of advection, we then immediately increase the decorrelation time scale 1 order of magnitude, which will lead to meaningful zooplankton distributions.

When we know the flow fields, the new LagrangianEulerian objective interpolation technique treats the samples as Lagrangian drifters. First, we calculate the Lagrangian trajectories of the samples based on the known flow fields by

$$
\mathbf{X}_{i}(t)=\int_{l_{i}}^{t} \mathrm{u}\left[\mathbf{X}_{i}(t), t\right] \mathrm{d} t
$$

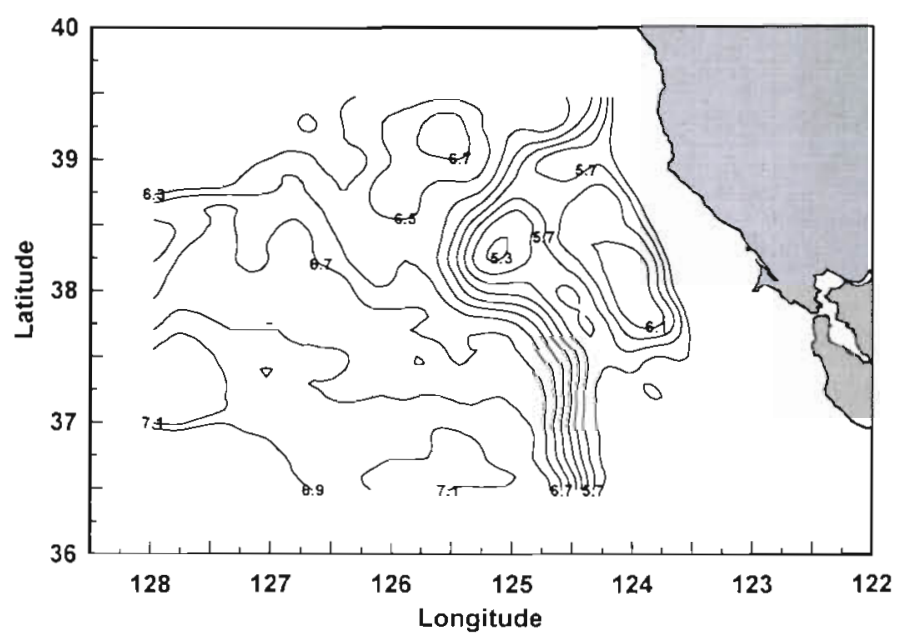

Fig. 1. The dynamic height at $25 \mathrm{~m}$ from the large-scale survey in the California Current region during June 6 to 28, 1993 
where $\mathbf{X}_{i}(t)$ is the trajectory of a sample obtained at $\left(\mathbf{x}_{i}, t_{i}\right)$. Because we only consider the advection process, the time can be reversed. In a given time interval $\Delta t$, the displacement of the drifter $\Delta \mathbf{X}_{i}$ can be simply estimated by

$$
\Delta \mathbf{X}_{i}=\Delta t\left[\mathbf{u}+\frac{\Delta t}{2}\left(\frac{\partial \mathbf{u}}{\partial t}+\mathbf{u} \nabla \mathbf{u}\right)\right]_{\left[\mathbf{X}_{i}(\ell, !)\right]}
$$

where is the notation of dot product of 2 vectors, and $\nabla$ is the spatial gradient operator.

The optimal estimate can then be written as

$$
\theta^{*}(\mathbf{x}, t)=\sum_{i=1}^{N} w\left[\mathbf{x}, \mathbf{X}_{i}(t), t, t_{i}\right] \varphi\left[\mathbf{X}_{1}(t), t_{1}\right]
$$

$\varepsilon(\mathbf{x}, t)^{2}=C(\mathbf{x}, \mathbf{x}, t, t)$

$+\sum_{i=1}^{N} \sum_{j=1}^{N} C\left[\mathbf{x}, \mathbf{X}_{i}(t), t, t_{i}\right] C\left[\mathbf{x}, \mathbf{X}_{j}(t), t, t_{j}\right] A^{-1}\left[\mathbf{X}_{i}(t), \mathbf{X}_{j}(t), t_{i}, t_{j}\right]$

where $w$ can be calculated based on Eqs. (6), (7) \& (8), i.e.

$$
\begin{array}{r}
w\left[\mathbf{x}_{1} \mathbf{X}_{i}(t), t_{1} t_{i}\right]=\sum_{j=1}^{N} C\left[\mathbf{X}_{1} \mathbf{X}_{j}(t), t, t_{j}\right] A^{-1}\left[\mathbf{X}_{i}(t), \mathbf{X}_{j}(t), t_{j}, t_{j}\right] \\
C\left[\mathbf{x}, \mathbf{X}_{i}(t), t_{,} t_{i}\right]=\left\langle<\theta(\mathbf{x}, t) \theta\left[\mathbf{X}_{i}(t), t_{i}\right]\right\rangle
\end{array}
$$

and

$$
\begin{aligned}
A\left[\mathbf{X}_{l}(t), \mathbf{X}_{j}(t), t_{i}, t_{j}\right] & =\left\langle\varphi\left[\mathbf{X}_{j}(t), t_{j}\right] \varphi\left[\mathbf{X}_{j}(t), t_{j}\right]\right\rangle \\
& =C\left[\mathbf{X}_{j}(t), \mathbf{X}_{j}(t), t_{j}, t_{j}\right]+E \delta_{i j}
\end{aligned}
$$

Because we have followed the trajectories of sample drifters, the advection time scale is removed from correlation functions. By doing this, we have increased the temporal correlation 1 order of magnitude. The decorrelation time scale is now determined by the diffusion and the plankton turnover time scales of $3 \times 10^{1}$ to $3 \times 10^{3} \mathrm{~d}$ and $10^{1} \mathrm{~d}$ respectively. Obviously new errors have been introduced into the analysis, which come from the estimate of flow fields. This also can be objectively analyzed.

The difference between this Lagrangian-Eulerian method and others is that the samples are not fixed at the sampling locations. The locations of samples move in the flow field as Lagrangian drifters. The spatiotemporal correlation is still Eulerian.

\section{APPLICATION TO PLANKTON DISTRIBUTION IN THE CALIFORNIA CURRENT REGION}

To more effectively elucidate the procedures of this Lagrangian-Eulerian technique, the application is limited to a horizontal 2-dimensional space even though the method itself is 3-dimensional. The plankton biomass was integrated in the upper $300 \mathrm{~m}$. By doing so, the effects of vertical current shear and diel vertical migration of plankton can be minimized (Huntley et al. 1995). Although out of the scope of this paper, differential advection and vertical migration are essential processes in determining plankton distributions and deserve further detailed study.

The California Current is fundamentally geostrophic (Huyer et al. 1991, 1994). The current jet and mesoscale eddies were drifting westward during our survey period at an approximate speed of $2 \mathrm{~cm} \mathrm{~s}^{-1}$ (Huyer et al. 1994). At this translation speed, the distance shift was approximately equal to $36 \mathrm{~km}$ in the survey period of $21 \mathrm{~d}$, which is much smaller than the advection distance of $360 \mathrm{~km}$ at a current velocity of $20 \mathrm{~cm} \mathrm{~s}^{-1}$. We make the assumption that the geostrophic flow is steady at the first approximation. We choose the averaged geostrophic current between 0 and 200 dbar and referred to 300 dbar as the mean flow field because most of the plankton are concentrated in the top $200 \mathrm{~m}$ (Huntley et al. 1995). The geostrophic flow field is produced from the objective analysis of CTD data (Huyer et al. 1994). We are aware of the errors produced by this approximation: (1) the error field of the estimated geostrophic flow, (2) the differential advection due to the vertical shear of currents, and (3) the ageostrophic components of the currents.

A detailed description of the plankton data can be found in Huntley et al. (1995). Briefly, an OPC was mounted on a SeaSoar (Chelsea Instruments, East Molesey, Surrey, UK), which vertically undulated between 0 and $300 \mathrm{~m}$ at a towing speed of $8 \mathrm{knots}$. The survey consisted of 13 meridional transects. During post data processing, OPC data were integrated over every upward or downward profiling (approximately $1 \mathrm{~km}$ ) as 1 independent measurement. The water volume filtered by the OPC over one of these profiles was approximately equal to $5 \mathrm{~m}^{3}$. Filtration of this amount

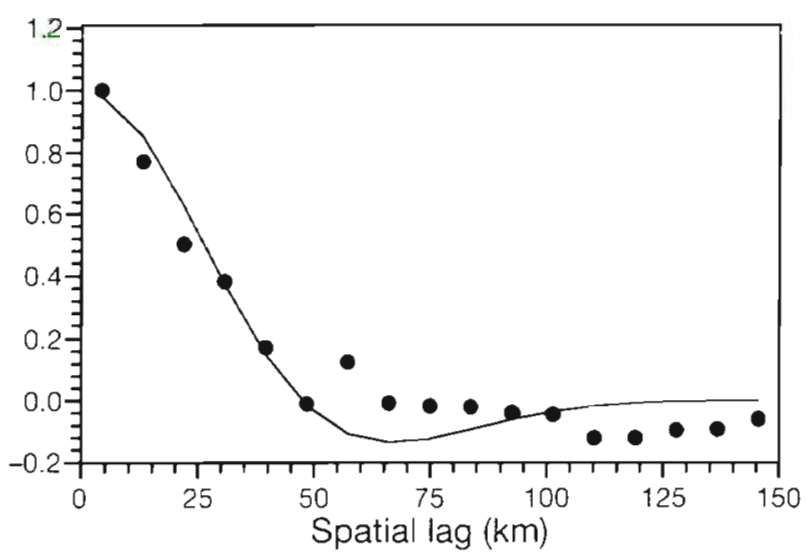

Fig. 2. Autocorrelation. The solid dots are the autocorrelation based on vertically integrated OPC data, and the solid line is the best-fit of Eq. (25) 
of water is required of animals of higher weight classes $\left(>10^{2} \mu \mathrm{g} \mathrm{C}\right)$ if they are fewer in number than $10 \mathrm{ani}$ mals $\mathrm{m}^{-3}$ (Huntley et al. 1995, Zhou \& Huntley 1997). The measurements were integrated into $0.1^{\circ}$ in meridional bins. The length of each bin is approximately equivalent to $10 \mathrm{~km}$. A spatio-autocorrelation of the OPC data shows that the first zero-cross is at $50 \mathrm{~km}$ and that the correlation is higher than $85 \%$ within a distance of $10 \mathrm{~km}$ (Fig. 2). This integration over a $10 \mathrm{~km}$ bin may eliminate the spatial scale of single species swarms, but it should not eliminate the dominant spatial features of plankton distributions of mixed species. Both mean and variance of zooplankton biomass were calculated in each bin for further use.

The displacement of each datum in $\Delta t$ marked by the sampled location and time $\left(\mathbf{x}_{\mathrm{i},} t_{1}\right)$ can be calculated based on Eq. (18) as

$$
\Delta \mathbf{X}_{l}=\Delta t\left[\mathbf{u}+\frac{\Delta t}{2} \mathbf{u} \cdot \nabla \mathbf{u}\right]_{\left.-\mid \mathbf{x}_{i}(t), t\right)}
$$

where we assumed that the flow is at quasi-steady state. Because the time can be reversed in the advection process, $\Delta t$ can be a positive or negative dependent of $t$, which represents the past or future relative to $t_{r}$. Thus the locations of all data can be calculated at a given time $t$.

In an ideal case, a 2-dimensional correlation should be performed based on the observed data. In our case, the resolution in the zonal direction is $0.25^{\circ}(\approx 25 \mathrm{~km})$. The zonal resolution of the data is poor for the 2-dimensional correlation calculation. The autocorrelation in Fig. 3 is in the meridional direction.

From the best fit of the autocorrelation (Huntley et al. 1995), we have

$$
C(r)=(1-r) \exp (-r)
$$

where $r$ is the normalized spatial distance between 2 locations. The spatial decorrelation scale determined by the first zero-cross in Fig. 3 is approximately equal to $50 \mathrm{~km}$. We modify this relation as

$$
r=\left\{\left(\frac{\Delta x}{L}\right)^{2}+\left(\frac{\Delta y}{L}\right)^{2}+\left(\frac{\tau}{T}\right)^{2}\right\}^{1 / 2}
$$

where $L$ is the spatial decorrelation scale of $50 \mathrm{~km}$, and $T$ is the temporal decorrelation scale. The decorrelation time scale can be estimated by
$L^{2} / A_{\mathrm{H}}$, where $A_{\mathrm{H}}$ is the horizontal diffusion coefficient. If $A_{\mathrm{H}}$ is approximately equal to $10^{5}-10^{7} \mathrm{~cm}^{2} \mathrm{~s}^{-1}$, the dispersion decorrelation time scale is larger than $28 \mathrm{~d}$. An estimate of this scale based on the zooplankton generation turnover time is approximately equal to $30 \mathrm{~d}$ (Huntley \& Boyd 1984, Mullin 1991). Thus, we chose the decorrelation time scale equal to $30 \mathrm{~d}$.

The survey was conducted from June 7 to 28, 1993. At any given time, the locations of samples were first calculated by Eq. (24), followed by the spatial objective interpolation as given by Eqs. (19) to (26). The estimated confidence levels determine the significance of interpolation. To describe the time-transient process, we choose an interpolated distribution of zooplankton at $t=0$ h on June 8,11,14,17, 20,23, and 26 in 1993.
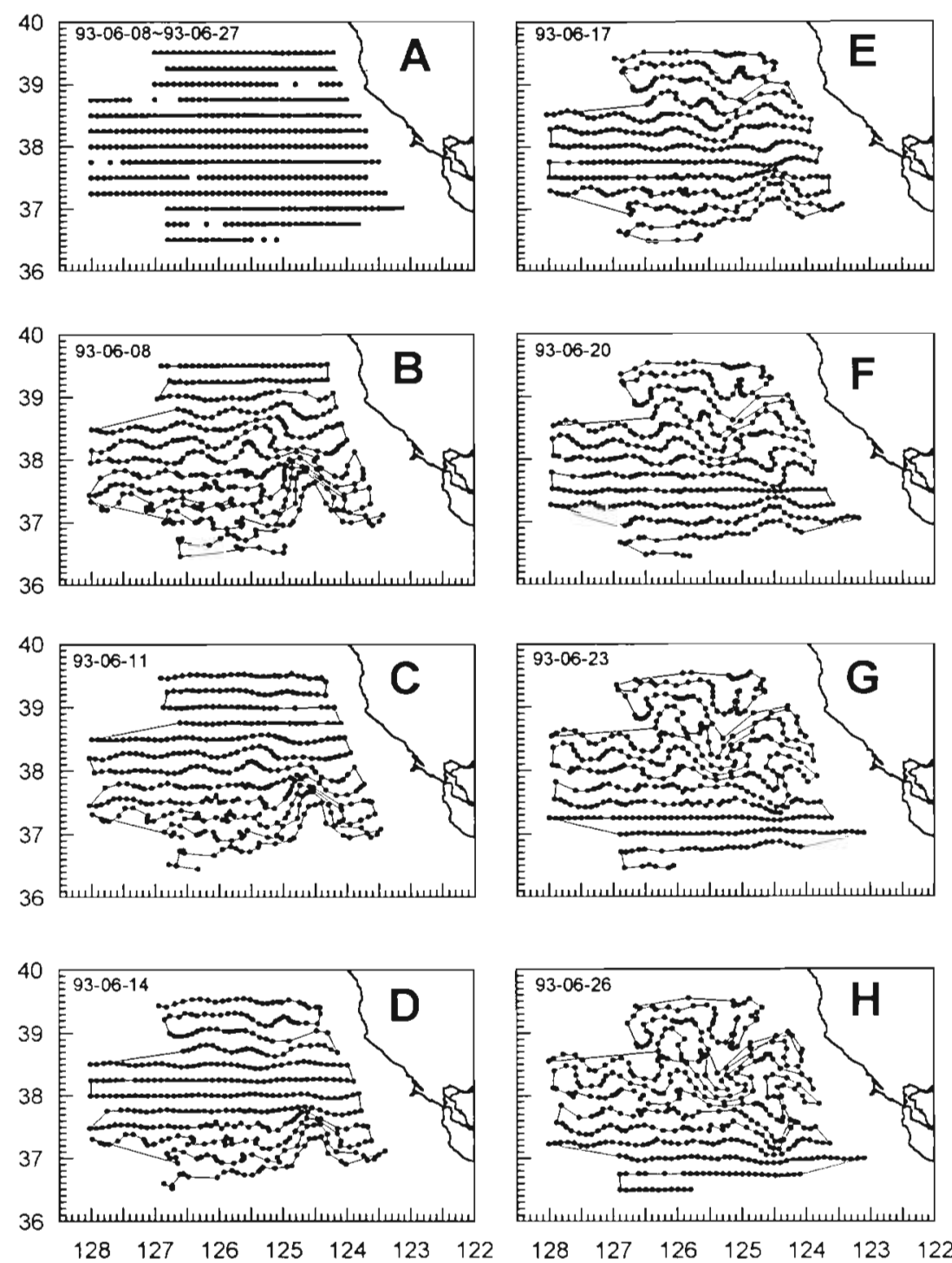

Fig. 3. Sample locations. (A) Original geographic locations of OPC samples and $(\mathrm{B}-\mathrm{H})$ traced locations of OPC samples as Lagrangian drifters by Eq. (17) at $0 \mathrm{~h}$ on the given day indicated on the upper-left corner of each panel 

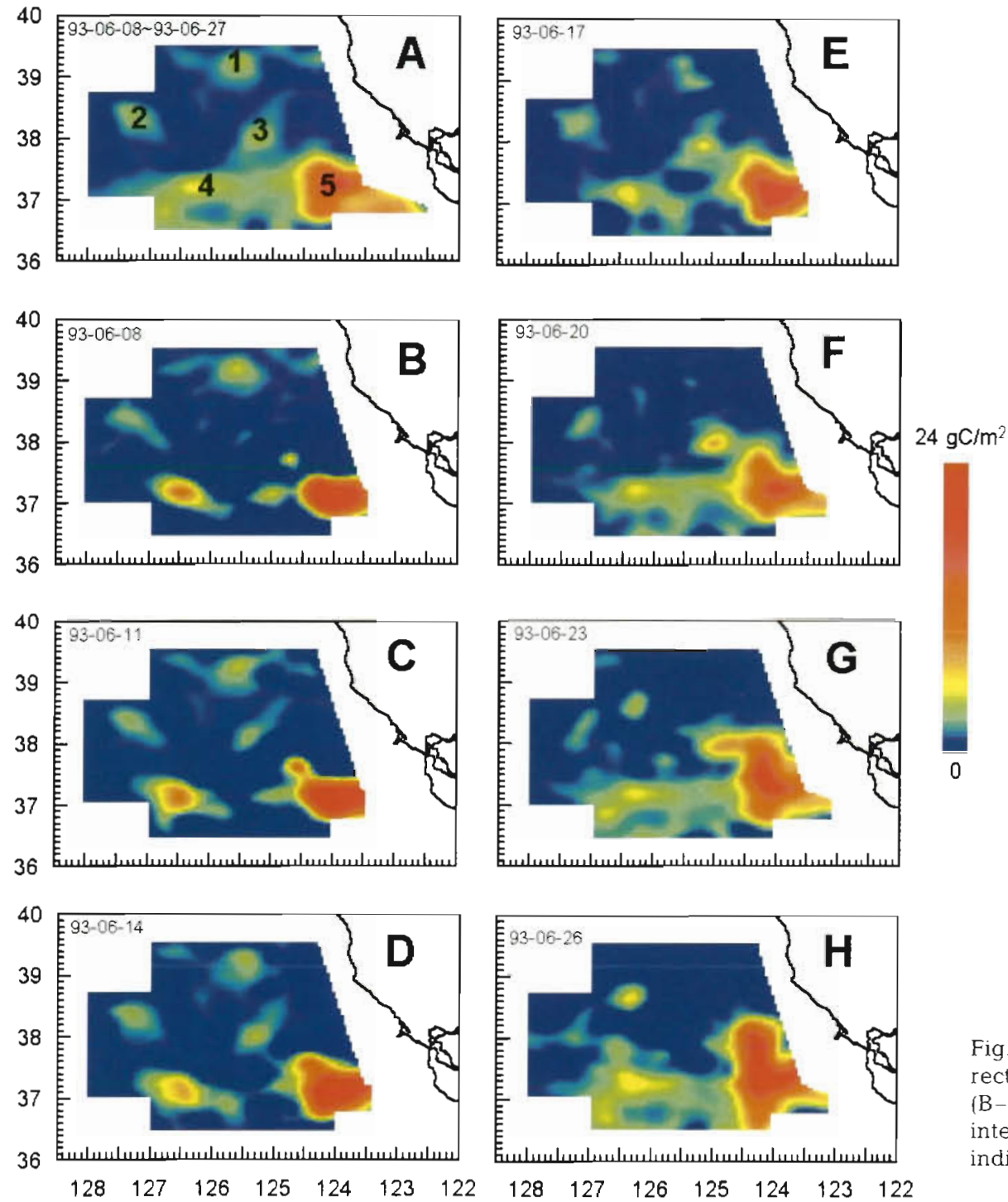

Fig. 4. Zooplankton distribution. (A) Direct interpolation of OPC samples and $(\mathrm{B}-\mathrm{H})$ Lagrangian-Eulerian objective interpolation at $0 \mathrm{~h}$ on the given day indicated on the upper-left corner of each panel

Fig. 3 shows sample locations and Figs. 4 \& 5 show the optimal estimates and the relative errors at the $95 \%$ confidence level of the plankton distribution, respectively.

\section{INTERPOLATED ZOOPLANKTON DISTRIBUTION}

Our survey in the California Current region revealed meso and large scale spatial features in zooplankton distributions (Fig. 4A). For convenience, the areas containing these features are marked as Areas 1 to 5: Area 1 includes an anticyclonic eddy and an offshore zooplankton maximum; Area 2 indicates meanders of currents and an offshore zooplankton maximum; Area 3 contains a mesoscale jet-cyclonic eddy system associated with the California Current and a zooplankton maximum centered in the jet; Area 4 is a region where currents are weak and zooplankton are distributed over most of this large area; and Area 5 shows the highest zooplankton maximum in our entire survey region.

The offshore maximum centered in the anticyclonic eddy in Area 1 has an error rate of less than $50 \%$ in the first half period of the survey. The disappearance of this maximum in the later period could have been caused simply by the time decorrelation of those measurements of high biomass in this anticyclonic eddy at the beginning of our survey. Because the secondary circulation within this eddy is at least 1 order of magnitude less than the mean flow, the zooplankton population in this eddy was nearly enclosed during the period of our survey. Thus, the decorrelation was caused by dispersion and population turnover

During our survey the offshore maximums in Areas 2 and 4 remained at a fair error level $(<50 \%)$, that indicated the existence and persistence of high zooplank- 
Fig. 5. Relative errors at $95 \%$ confidence. (A) No statistical results and $(\mathrm{B}-\mathrm{H})$ objective error mapping at $0 \mathrm{~h}$ on the given day indicated on the upper-left corner of each panel
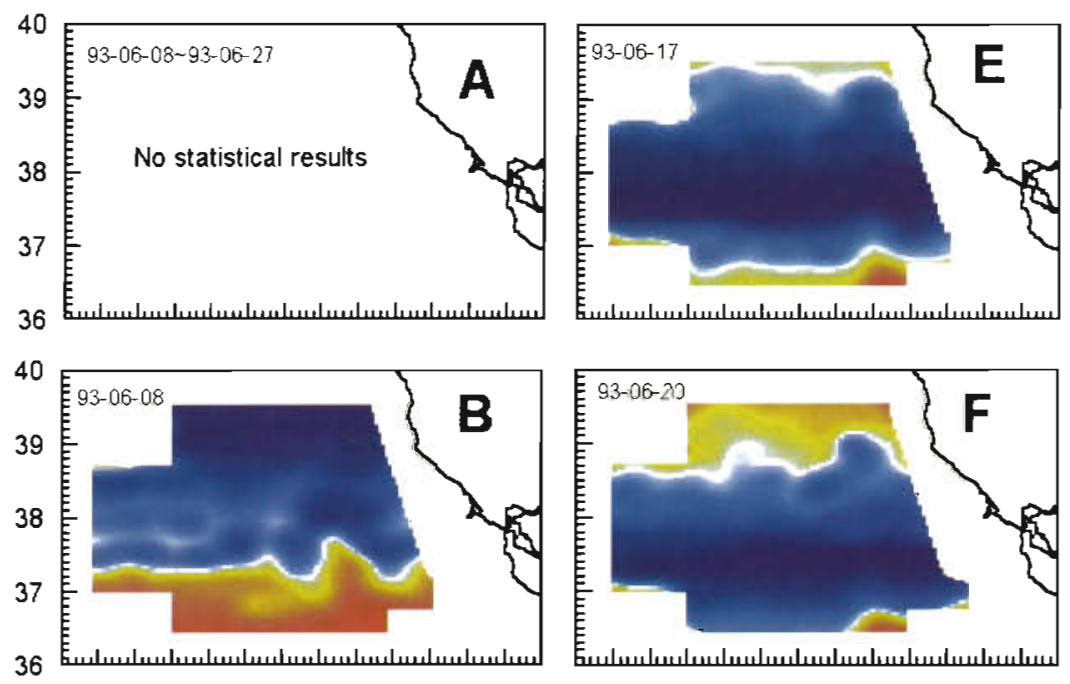

$100 \%$
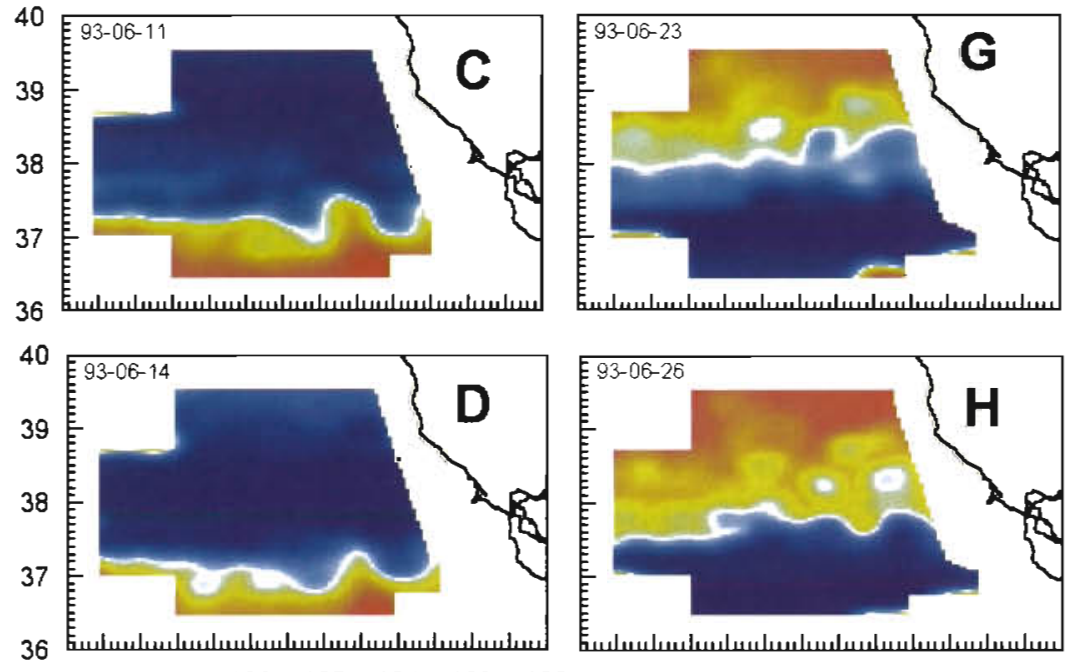

$\begin{array}{llllllllllllll}128 & 127 & 126 & 125 & 124 & 123 & 122 & 128 & 127 & 126 & 125 & 124 & 123 & 122\end{array}$ ton biomass distribution in the offshore regions. These maximums were associated with meanders of currents instead of eddies. These samples in Areas 2 and 4 were taken in the middle of the survey, so that the time lags between interpolation and sampling times were relatively small.

One of the robust results from this LagrangianEulerian interpolation is the trajectory of a zooplankton maximum measured in Area 3 , which was centered in the California Current. This maximum was advected southwestward around the mesoscale eddy, eastward to the shore, and then southward. It converged with the maximum in Area 5. These results show that in the California Current region the zooplankton distribution is very transient.

The nearshore zooplankton maximum in Area 5 had the highest biomass in our survey region. The downstream advection of zooplankton from the north by the California Current is apparently one of the processes producing this maximum. Meanwhile a nearshore anticyclonic eddy formed between the nearshore current and the California Current. The eddy's current advected zooplankton in this maximum northward, and then recirculated southward to join the nearshore current and the California Current.

\section{DISCUSSION}

\section{Time-dependent distribution of plankton}

If we calculate the past and future of a Lagrangian trajectory of a sample we can assume a reasonable time scale of decorrelation. Samples can be pooled from all available observations made during the entire survey. Objective analysis allows us to weigh the information by Eqs. (25) \& (26) according to lags over space and time. Samples that fall in the jet region will be 
quickly advected downstream, and those trapped in eddies will remain near the original locations. On June 8 , significant samples were located in the northern part of our survey area and a few samples were trapped in enclosed eddies in the southern part (Fig. 4). In contrast, on June 26, significant samples were located in the southern part. In the middle of the survey on June 14 and 17 , significant samples were more uniformly distributed over whole area than those in the beginning and end of the survey.

As we expected, advection is most important in areas of strong currents, such as the California Current, nearshore currents, and the northward current between the California Current and nearshore currents. The advection effect on the distribution of plankton has been one of the major obstacles in the studies of an ecosystem in which there are strong currents. Combining samples in the Lagrangian way removes the advective bias from samples, and provides a time-dependent plankton distribution.

Temporal variation of plankton is determined by advection, dispersion, behaviors and population growth of plankton. These processes are generally included and coupled in our samples. To count the advection in the Lagrangian-Eulerian interpolation separates the advection process from the others. The interpolated data can then be used to further analyze the processes of behaviors and population dynamics.

\section{Improving confidence}

The errors of interpolated distributions are greater than $50 \%$ in the southern portion of the survey region during the early survey period (June 8 to 14, 1993) (Fig. 5). This is caused by the lags between the interpolation and locations-times of samples. Similarly, the error is greater than $50 \%$ in the northern portion of the survey region during the later survey period (June 20 to 26 , 1993). The overall best estimates are given at the middle of the survey area on June 17 where, except for a small area in the south, the error is less than $50 \%$. It is logical that an estimate has a smaller error where the location-time of the estimate is closer to the locationstimes of surrounding samples. This error analysis indicates that our survey in the California Current region provided spatial and temporal distributions at a reasonable error level $(50 \%)$ only in the middle of the survey.

The principle for improving the accuracy of interpolation is to increase the correlation between the interpolation and surrounding samples in space and time. Reduction of the survey time by increasing sampling speed can improve the confidence interval. Elimination of any short scales in space and time improves the correlation too. Additionally, new scales may be intro- duced during the elimination; for example, how long can we actually trace a sample-drifter? If this time scale were less than that of advection, we would not benefit by using Lagrangian-Eulerian analysis. In this case the time scale is best determined by the dispersion process (Davis 1991), which is approximately equal to $30 \mathrm{~d}$.

The time scale of decorrelation due to generation turnover will also reduce the confidence of interpolation. Increasing the sampling speed can improve the confidence. As the sampling speed has been limited by our existing technology, consideration of population dynamics may be a more practical way to improve the interpolation confidence interval. Assuming that $Z$ is the abundance or biomass of one plankton component classified by species-stages or simply by weight, and the current fields are defined by velocities $\mathbf{u}(\mathbf{x}, t)$, the plankton distribution and productivity can be determined by the advection and population dynamics processes, i.e.

$$
\frac{\partial Z}{\partial t}+\nabla(\mathbf{u} Z)=\left(\frac{\mathrm{d} Z}{\mathrm{~d} t}\right)_{p}
$$

In Eq. (27), the second term on the left represent the advection of plankton, and $(\mathrm{d} Z / \mathrm{d} t)_{p}$ on the right represents the rate of change in plankton abundance or biomass associated with population dynamics and behaviors. Population dynamics of plankton can be modeled in 2 ways. One is based on species-stage structure of zooplankton (Ohman 1988, Huntley et al. 1994), and the other is based on biomass spectra of plankton (Zhou \& Huntley 1997). The behavior of zooplankton can be closed by the bio-continuum theory (Zhou \& Huntley 1996).

\section{Guidance in designing survey grids}

This study in the region of the California Current showed that the synoptic scale of plankton distribution is on the order of $50 \mathrm{~km}$ (Huntley et al. 1995). The variation of plankton at this scale reflects their population dynamics at generation time scales of weeks and months (Haury et al. 1978). In order to study the population dynamics of plankton in a given feature, a time series of samples during the generation time scale is needed to analyze population dynamics rates. When advection has a time scale of 3 to $10 \mathrm{~d}$, repeat sampling is a very difficult task. A mesoscale zooplankton feature can be advected out of a mesoscale survey area during the survey period of 3 to $10 \mathrm{~d}$.

This Lagrangian-Eulerian interpolation provides a solution for eliminating the advection effect. Repeated samples in a given area can be used to reconstruct a time series of spatial distributions as we have demonstrated. It is more important that this method can be 
used to theoretically guide the survey and the sampling strategies. Given the flow field, decorrelation scales and measurement errors, the survey error fields can be directly estimated from the survey design, so that an optimal survey grid can be determined which will permit the good interpolation of spatial and temporal distribution of zooplankton from samples.

\section{The link between field observations and modeling}

In past decades, the modeling of spatial and temporal distributions of plankton has proven to be a useful tool for understanding some processes (Fasham et al. 1990, Franks \& Chen 1996). Verification of zooplankton modeling results requires a time series of zooplankton spatial distribution. Such data sets are very rare as our existing sampling methods cannot directly provide such synoptic spatial coverage. Thus, most models remain in theoretical cases and laboratory experiments. This Lagrangian-Eulerian interpolation provides a time series of zooplankton distributions and their error fields, and can directly confirm results of a model. This method is a direct link between field observations and modeling.

\section{CONCLUSION}

Recognizing the advection of passive marine plankton, a new Lagrangian-Eulerian interpolation tech- nique has been developed based on the GaussMarkov theorem explicitly showing the result in statistical estimation theory. Because we remove the decorrelation time scale due to advection, the error of interpolation is much improved. Based on statistical assumptions, this method yields at every point $(x, y$, $z, t)$ an estimate which is optimal in the sense of the least-squares error.

This Lagrangian-Eulerian interpolation can be employed to analyze space-time dependent plankton samples and produces the spatial distribution of plankton at a given time. The application of this technique to the plankton data obtained by an OPC in the California Current during June 1993 revealed not only plankton maximums associated with physical features, but also associated transports and exchanges of plankton with currents and eddies. The analysis of differential advection due to velocity shear, secondary circulation, and vertical migration behavior is definitely required to further understand the temporal and spatial distribution plankton in the California Current region.

Acknowledgements. This research was supported by Office of Naval Research grant number N00014-92-J-1618 and NSF grant OCE97-11261. Special thanks are due to Y. Zhu for data processing and programming supports, Dr M. E. Huntley and $\mathrm{K}$. Whittaker for comments and encouragement. This is contribution No. 15 of the Simon J. Poole Institute of Theoretical and Observational Biology, and contribution 108 of the U.S. GLOBEC program, funded jointly by NSF and NOAA

Appendix 1. Glossary of terms and math symbols

\begin{tabular}{|c|c|c|c|}
\hline$A\left(\mathbf{x}_{1}, \mathbf{x}_{i}, t_{1} t_{j}\right)$ & Matrix of correlation functions & $\varepsilon$ & Measurement error \\
\hline$A^{-1}\left(\mathbf{x}_{,} \mathbf{x}_{n} t, t_{i}\right)$ & Inverse matrix of $A$ & $\varphi$ & Measurement of $\theta$ \\
\hline$A_{\mathrm{H}-\mathrm{H}}$ & Horizontal diffusivity & $\theta$ & Scale field \\
\hline$C\left(\mathbf{x}, \mathbf{x}_{i}, t_{i} t_{i}\right)$ & Eulerian spatiotemporal correlation & $\theta^{*}$ & Estimate of $\theta$ \\
\hline$L, L_{x}, L_{y}, L_{z}$ & $\begin{array}{l}\text { Spatial scales of zooplankton } \\
\text { distribution }\end{array}$ & $\begin{array}{l}\tau \\
\mathbf{a} \cdot \mathbf{b}\end{array}$ & $\begin{array}{l}\text { Time lag } \\
\text { Dot product of vectors } \mathbf{a} \text { and } \mathbf{b}\end{array}$ \\
\hline$L_{\mathrm{s}}$ & Survey resolution & $\delta_{i j}$ & Kroenecker delta \\
\hline $\mathrm{r}$ and $(\Delta x, \Delta y, \Delta z)$ & $\begin{array}{l}\text { Spatial lag or distance between } 2 \\
\text { Eulerian locations }\end{array}$ & i & \\
\hline$t$ & Time & $\int_{a}$ & Finite integral \\
\hline$\Delta t$ & Time interval & $\partial$ & \\
\hline$T_{s}$ & Survey time & $\frac{\partial}{\partial t}$ & Partial derivative \\
\hline$T, T_{z}$ & $\begin{array}{l}\text { Time scale of zooplankton } \\
\text { distribution }\end{array}$ & $\nabla$ & Gradient operator defined as $\nabla=$ \\
\hline $\mathbf{u}$ & Eulerian velocities & & $\frac{\partial}{\partial x} \hat{x}+\frac{\partial}{\partial y} \hat{y}+\frac{\partial}{\partial z} \hat{z}$ where $(\hat{x}, \hat{y}, \hat{z})$ \\
\hline$w\left(\mathbf{x}, \mathbf{x}_{i}, t_{,} t_{i}\right)$ & Weight function & & are the unit direction vectors \\
\hline$x$, and $(x, y, z)$ & $\begin{array}{l}\text { Eulerian coordinates } \\
\text { Larangian trajectory of a sample }\end{array}$ & $\Sigma$ & Sum \\
\hline $\begin{array}{l}\mathbf{X}(t) \\
\Delta \mathbf{X}(t)\end{array}$ & $\begin{array}{l}\text { Lagrangian trajectory of a sample } \\
\text { Lagrangian displacement of a } \\
\text { sample }\end{array}$ & $\langle\cdot\rangle$ & Ensemble average \\
\hline
\end{tabular}




\section{LITERATURE CITED}

Bretherton FP, Davis RE, Fandry CB (1976) A technique for objective analysis and design of oceanographic experiments applied to MODE-73. Deep-Sea Res 23: $559-582$

Carter EF, Robinson AR (1987) Analysis models for the estimation of ocean fields. J Atmos Oceanic Tech 4:49-74

Clancy RM (1983) The effect of observational error correlations on objective analysis of ocean thermal structure. Deep-Sea Res 30:985-1002

Davis JC (1986) Statistics and data analysis in geology. John Wiley \& Sons, New York

Davis RE (1991) Observing the general circulation with floats. Deep-Sea Res (Suppl 1) 38:\$531-\$571

Dentsch CV, Journel AG (1992) GSLB: geostatistical software library and user's guide. Oxford University Press, Oxford

Fasham MJR, Ducklow HW, McKelvie SM (1990) A nitrogenbased model of plankton dynamics in the oceanic mixed layer. J Mar Res 48:591-639

Franks PJS, Chen C (1996) Plankton production in tidal fronts: a model of Georges Bank in summer. J Mar Res 54: $631-651$

Gandin LS (1965) Objective analysis of meteorological fields. Gidrometeorizdat, Leningrad

Gill AE (1982) Atmosphere-ocean dynamics. Academic Press, London

Haury LR, McGowan JA, Wiebe PH (1978) Patterns and processes in time-space scales of plankton distributions. In Steele JH (ed) Spatial patterns in plankton communities. Plenum Press, London, p 277-328

Huntley ME, Boyd C (1984) Food-limited growth of marine zooplankton. Am Nat 124:455-478

Huntley ME, Niiler PP (1995) Physical control of population dynamics in the Southern Ocean. ICES J Mar Sci 52: $457-468$

Huntley ME, Zhou M, Lopez MDG (1994) Calanoides acutus in Gerlache Strait, Antarctica II. Solving an inverse prob-

Editorial responsibility: Otto Kinne (Editor),

Oldendorf/Luhe, Germany lem in population dynamics. Deep-Sea Res II 41:209-227

Huntley ME, Zhou M, Nordhausen W (1995) Mesoscale distribution of zooplankton in the California Current in late spring, observed by Optical Plankton Counter. J Mar Res 53:647-674

Huyer A, Kosro PM, Fleischbein J, Ramp SR, Stanton T, Washburn L, Chavez FP, Cowles TJ, Pierce SD, Smith RL (1991) Currents and water masses of the coastal transition zone off northern California, June to August, 1988. J Geophys Res 96:14809-14831

Huyer A, Barth JA, Kosro PM, Smith RL (1994) Surveys of the California Current: large-scale coverage with mesoscale resolution. EOS 75:140-140

Isaaks EH, Srivaslava $M$ (1989) An introduction to applied geostatistics. Oxford University Press, New York

Liebelt TB (1967) An introduction to optimal estimation. Addison-Wesley, Reading, MA

Mooers CNK, Robinson AR (1984) Turbulent jets and eddies in the California Current and inferred cross-shore transports. Science 223:51-53

Mullin MM (1991) Spatial-temporal scales and secondary production estimates in the California Current ecosystem In: Sherman K, Alexander LM, Gold BD (eds) Food chains, yields, models, and management of large marine ecosystems. Westview Press, Boulder, p 165-191

Ohman MD (1988) Predator-limited population growth of the copepod Pseudocalanus sp. J Plankton Res 8:673-713

Paduan JD, Niiler PP (1993) Structure of velocity and temperature in the northeast Pacific as measured with Lagrangian drifters in fall 1987. J Phys Oceanogr 23:585-600

Steele JH, Henderson EW (1992) A simple model for plankton patchiness. J Plankton Res 14:1397-1403

Zhou M, Huntley ME (1996) The principle of biological attraction, demonstrated by the bio-continuum theory of zooplankton patch dynamics. J Mar Res 54:1017-1037

Zhou $M$, Huntley ME (1997) A population dynamics theory of plankton based on biomass spectra. Mar Ecol Prog Ser 159:61-73

Submitted: November 6, 1997; Accepted: September 10, 1998 Proofs received from author(s): November 11, 1998 